Revue des patrimoines

\title{
Objets de grève, un patrimoine militant
}

\section{Jean-Charles Leyris}

\section{OpenEdition}

\section{Journals}

Édition électronique

URL : http://journals.openedition.org/insitu/3044

DOI : 10.4000/insitu.3044

ISSN : 1630-7305

\section{Éditeur}

Ministère de la culture

\section{Référence électronique}

Jean-Charles Leyris, "Objets de grève, un patrimoine militant », In Situ [En ligne], 8 | 2007, mis en ligne le 01 mars 2007, consulté le 19 avril 2019. URL : http://journals.openedition.org/insitu/3044 ; DOI : 10.4000/insitu.3044

Ce document a été généré automatiquement le 19 avril 2019.

\section{(2) $\oplus \Theta \Theta$}

In Situ Revues des patrimoines est mis à disposition selon les termes de la licence Creative Commons Attribution - Pas d'Utilisation Commerciale - Pas de Modification 4.0 International. 


\title{
Objets de grève, un patrimoine militant
}

\author{
Jean-Charles Leyris
}

Remerciements particuliers à Jean-Luc Moulène, Françoise Bosman (directrice des ANMT), Catherine Jakubowski (secrétaire de documentation aux ANMT), Christian Hottin (Ministère de la culture et de la communication, chef de la mission ethnologie) et Noëlle Gérôme pour leur contribution à l'écriture de ce texte.

1 Les Archives Nationales du Monde du Travail (Roubaix) ${ }^{1}$ conservent, dans dix boîtes d'archives, une série d'objets d'apparence hétéroclite : petites bouteilles de parfum en plastique, souliers d'enfants, écharpe rouge, bobines de fils, tee-shirts imprimés et même des billets de banque factices, etc. Collectés en 2003, ils constituent pourtant un ensemble étonnant d'objets dits “ de grève". Généralement fabriqués par des ouvriers grévistes pendant des périodes d'occupation d'usine, ils se distinguent des produits manufacturés courants par leur forme, le plus souvent singulière, par les conditions de leur conception et fabrication, ainsi que par leur fonction (fig. $\mathbf{n}^{\circ} \mathbf{1}$ ). 


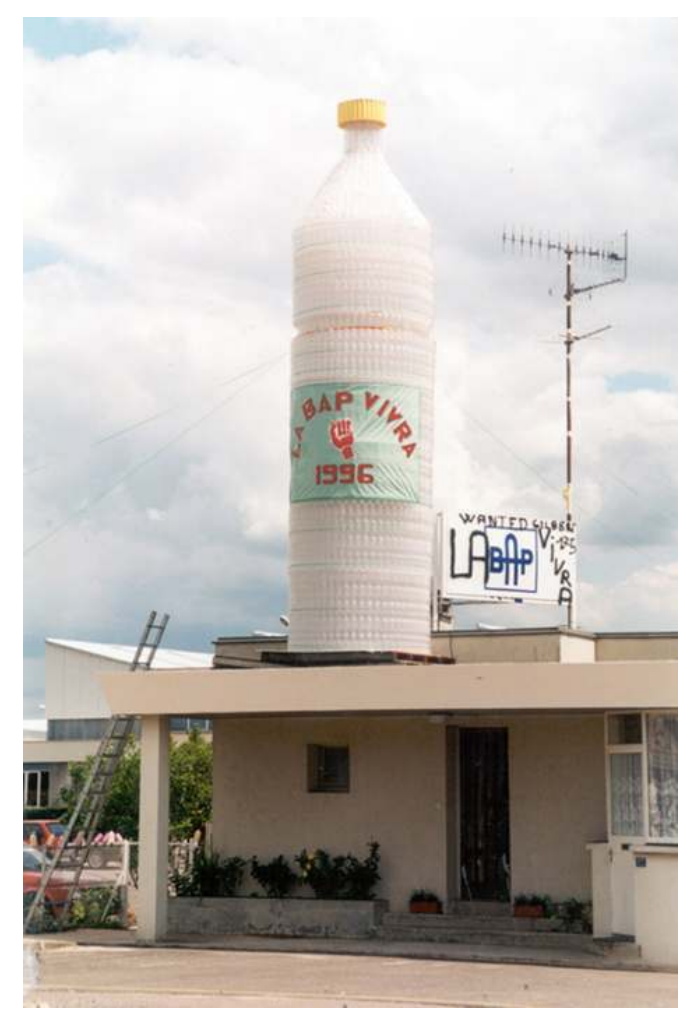

Chevigny-Saint-Sauveur (Côte d'Or). Le 27 juillet 1996. Présentation de la plus grosse bouteille plastique en PVC du monde. Manifestation hostile du personnel aux projets de fermeture de l'usine Bourgogne Applications Plastiques. Provenance : Archives Nationales du Monde du Travail, fonds Jean-Luc Moulène (cote 2003 025)

(c) Jean-Luc Moulène - ADAGP, 1999.

Objets produits pour faire connaître la lutte sociale ou la financer, ils constituent aussi un témoignage, côté métier, sur des savoir-faire professionnels et, côté militance, sur la gestion et le sens des conflits du travail où la contestation se mêle à la créativité dans les modes de manifestation. Ils sont aussi un reflet d'une époque, la fin des années 1970 et le début des années 1980, marquée par une crise industrielle et sociale profonde dont les traces sont encore tangibles.

Même si, aujourd'hui, les outils de la manifestation sociale ont pour partie changé (les fermetures d'usine se soldant parfois par des actions spectaculaires comme des menaces d'explosion ou de pollution), ces objets constituent une archive particulière mais précieuse venant utilement compléter d'autres fonds d'archives de forme plus classique : archives écrites ou audiovisuelles d'organisations syndicales, de militants politiques ou de comités d'entreprises. En quoi, cependant, peuvent-ils être classés sous la bannière de "patrimoine industriel "? Héritage du monde ouvrier en lutte, ce patrimoine matériel est aussi le fruit indirect de l'activité industrielle et de ses vicissitudes sociales. Néanmoins, ces objets ne sont pas directement industriels ou industrialisés, dans le sens où ils ne sont pas habituellement produits par l'entreprise et qu'ils ne sont pas non plus fabriqués et distribués en série pour satisfaire une consommation de $\operatorname{masse}^{2}$ (fig. $\mathbf{n}^{\circ} \mathbf{2}$ ). 


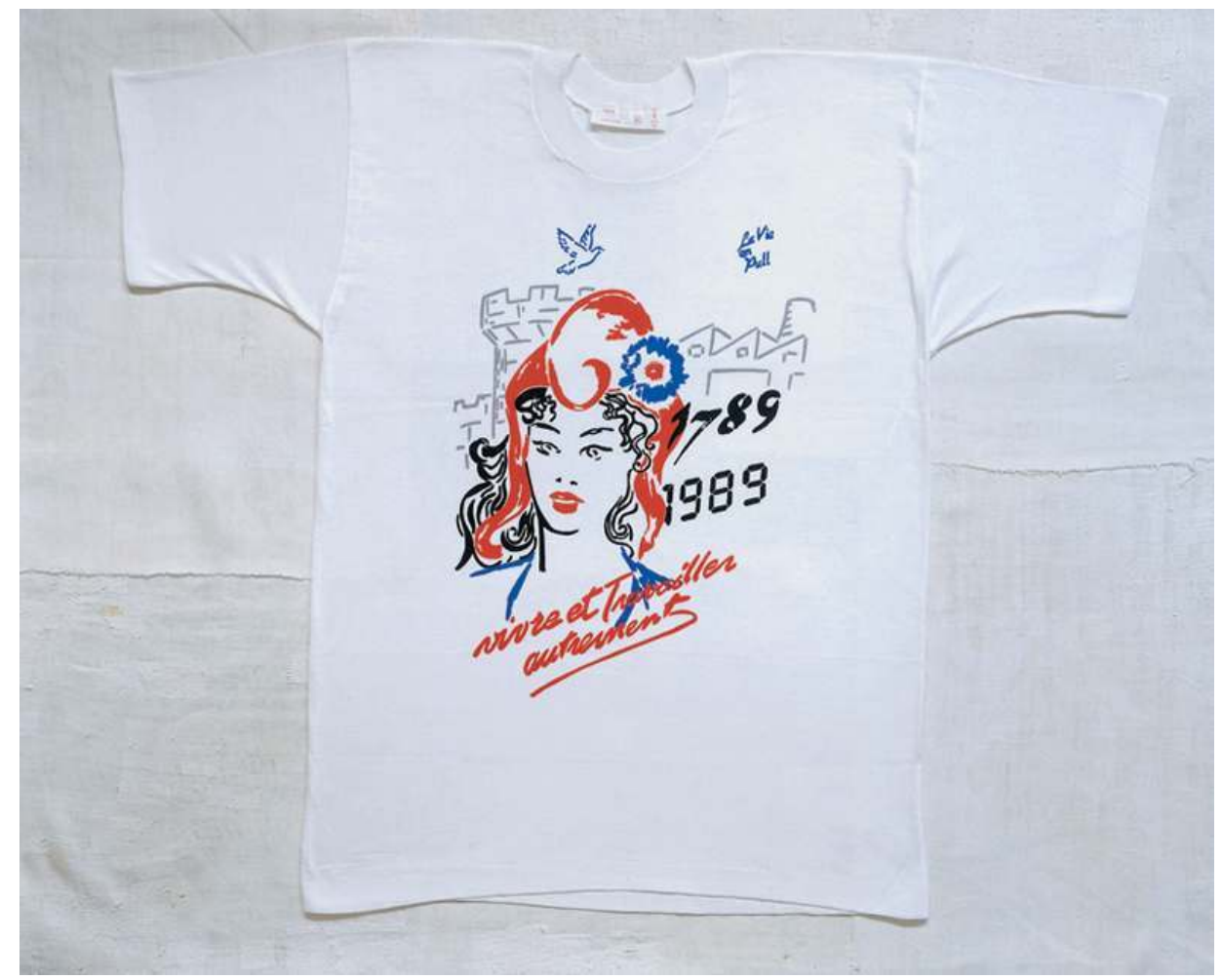

Le T-Shirt 1789-1989. T-shirt en coton sérigraphié La Vie en Pull. Roanne (Loire). Collection Printemps 1989. Courtesy galerie Chantal Crousel, Paris

(c) Jean-Luc Moulène - ADAGP, 1999.

4 La notion de patrimoine industriel s'est progressivement étendue, comme le rappelle Nicole Dorion", à celle d'objet industriel : “Les spécialistes de l'archéologie industrielle s'intéressaient surtout aux bâtiments. Puis, peu à peu, la notion de patrimoine industriel s'est élargie, à mesure que des spécialistes de différentes disciplines s'y sont intéressés. En ce qui concerne l'objet industriel, son intérêt réside dans le fait qu'il constitue un élément important supplémentaire pour la compréhension du genre de vie d'un peuple [...]. Aussi est-il nécessaire de remettre l'objet industriel dans son contexte historique, d'en situer l'invention, d'en voir l'évolution ou la transformation et s'il y a lieu, la disparition, car un objet n'a de sens du point de vue culturel que par la signification que lui donnent les individus de la société d'où il est issu ". Si la notion d'objet industriel a d'abord concerné les dispositifs de production de grande dimension, puis des machines de taille parfois plus modeste, nous sommes loin ici, avec les objets de grève, d'une définition standard du patrimoine, et même de l'industrie. Certes produits en usine, avec l'outillage utilisé d'ordinaire pour la fabrication, par les ouvriers de l'entreprise, ils sont issus d'un temps particulier (celui de la grève et donc d'un mode de fonctionnement de l'entreprise perturbé) et d'un mode d'organisation et de mise en œuvre du travail inhabituels. Sauf dans de rares cas, ils ne sont produits qu'en petites séries, dans un geste artisanal valorisant le savoir-faire et le plaisir du métier ${ }^{4}$ (fig. $\mathbf{n}^{\circ} 3$ ). 


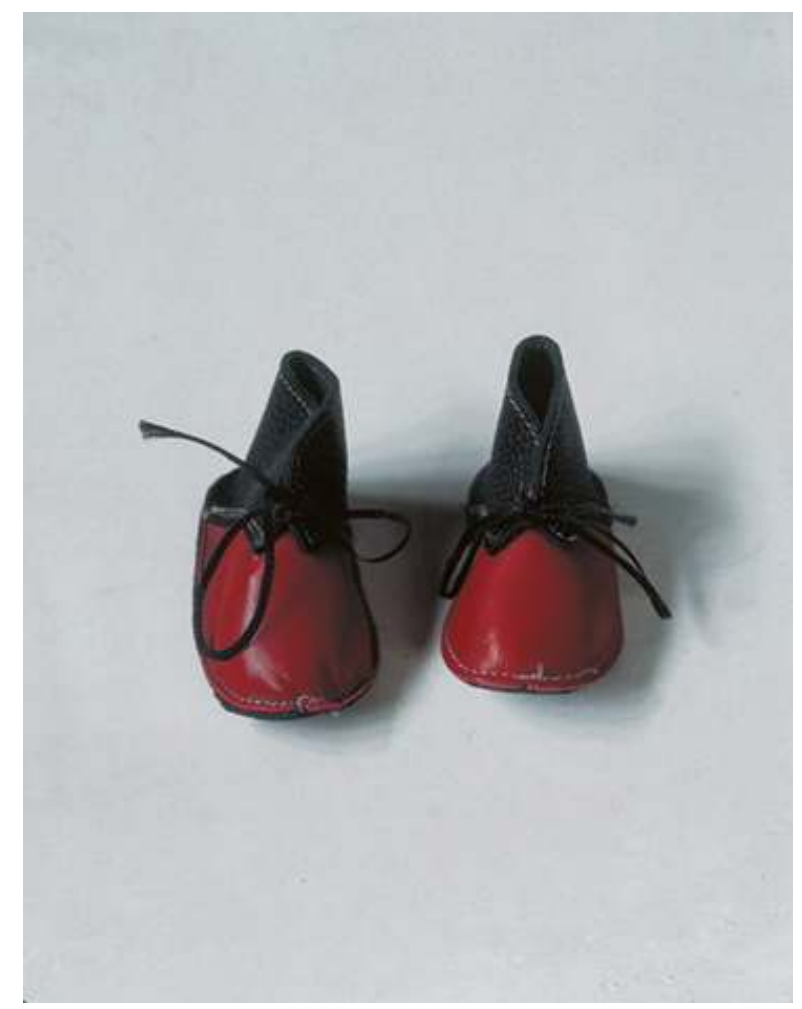

Les souliers de la lutte. Souliers couleurs " foot " rouge et noir. Myrys. Limoux (Aude). Courtesy galerie Chantal Crousel, Paris

(c) Jean-Luc Moulène - ADAGP, 1999.

$5 \quad$ Figures militantes, ces chaussures, écharpes et autres accessoires où pourrait se lire toute une culture populaire d'une époque révolue, ont aussi été, et c'est une autre de leurs particularités, transformés en œuvre d'art par l'intervention de Jean-Luc Moulène, artiste contemporain qui les a collectés, photographiés puis exposés. C'est d'ailleurs par ce biais que le grand public a pu avoir connaissance de ces objets de grève (connaissance à la fois des objets eux-mêmes et de leur double photographique) : les photographies, achetées par le Centre Georges Pompidou, font partie des collections nationales et sont exposées dans l'espace du musée dédié aux collections permanentes. La complexité de ces objets, d'appartenance à la fois militante, industrielle et artistique, traités d'un point de vue archivistique, va nous conduire d'une part à tenter de les décrire le plus précisément possible, d'autre part à mettre au jour les différents regards portés sur ces objets.

\section{Altérations}

6 Les objets dont il est question ici s'apparentent à première vue à des objets industrialisés courants : une manivelle de vélo, une poêle à frire, un jeu de société (fig. $\left.\mathbf{n}^{\circ} 4\right)$, un paquet de cigarettes, etc. 


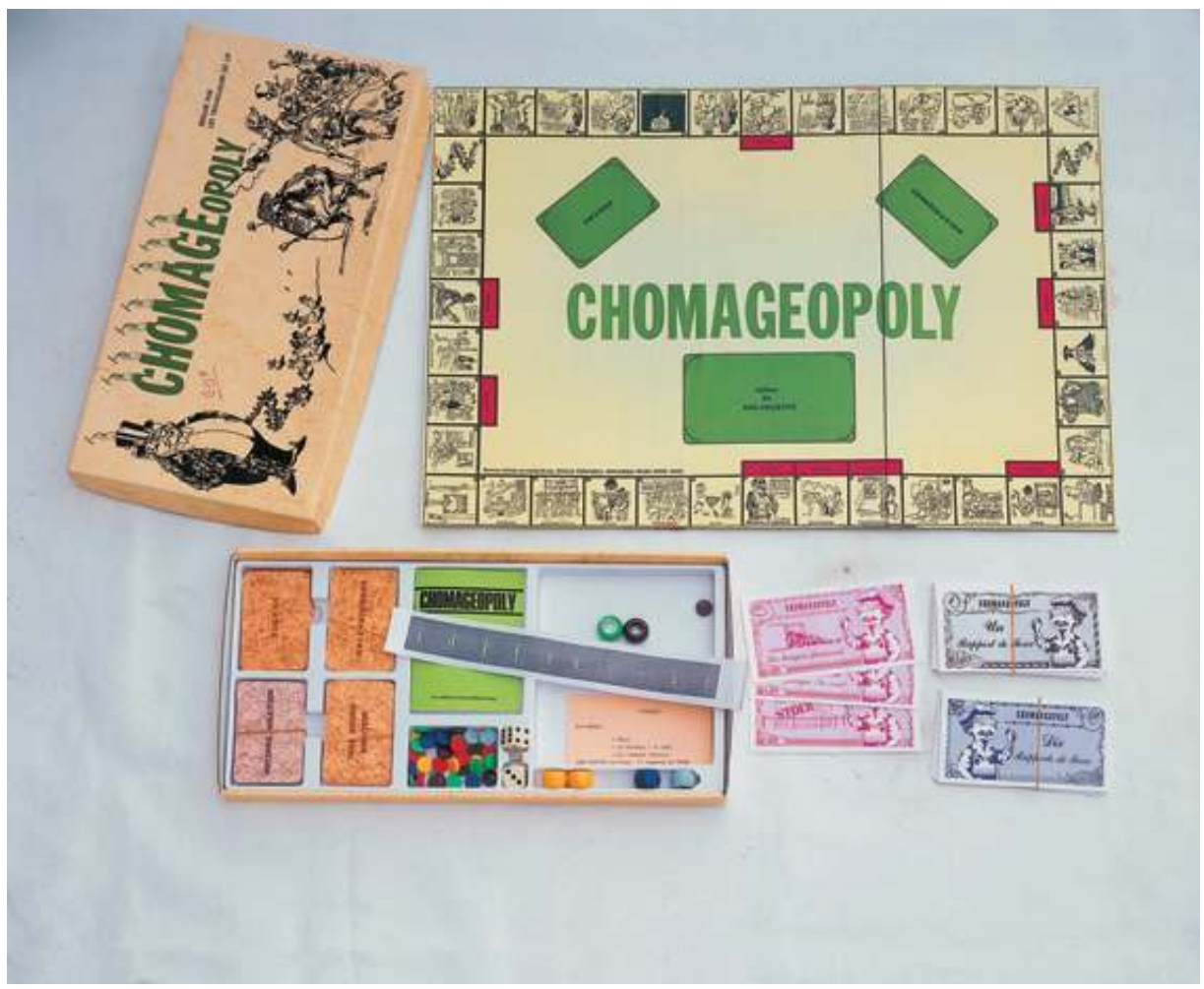

Chomageopoly. Lip, 2e conflit. Jeu de Monopoly (inventé par des chômeurs américains dans les années 1930) réalisé par les travailleurs de Lip. 42 × 21,3 cm, jeu : 40,5 × 59 cm. (Collection Michel Pelleteret). Courtesy galerie Chantal Crousel, Paris

(c) Jean-Luc Moulène - ADAGP, 1999.

Ils revêtent pourtant une singularité à la fois formelle et fonctionnelle. Des indices visibles nous mettent sur la voie: ainsi, au dos de la poêle peut-on lire en son centre “Relaxe " et sur sa circonférence " Emploi, solidarité, liberté, justice ". L'Herald Tribune du 5 juin 1987 ne présente que des rectangles blancs à la place des photographies qui auraient dû y être reproduites. Le Monopoly devient, au centre du plateau de jeu, le Chomageopoly. Le billet SNCF annonce sur son recto "Dernier billet avant augmentation ".

Des incongruités, des étrangetés, se glissent dans la banalité des objets tant consommés. Le monde des objets usuels se dérobe pour laisser place à des figures décalées qui nous interrogent sur notre vision du monde. Que se passe-t-il? Jean-Luc Moulène définit volontiers les objets de grève qu'il a collectés comme " des objets de production altérés " Ce qui signifie que quelque chose du processus normal de production s'est enrayé ou, plus précisément, a été volontairement grippé. Ce qui a changé, ce sont les conditions dans lesquelles ces objets ont été fabriqués. Et, plus que cela, ce qui a été perturbé, transformé, c'est la façon dont on a décidé de produire, dont on a conçu l'objet, par qui, avec quels moyens, à quelle fin: toute la chaîne industrielle remise en cause et reformulée, réadaptée à d'autres besoins pour un autre mode d'appropriation et de transmission de l'objet (le mot " consommation" serait ici impropre).

“Objets de grève : objet produit à des fins manifestes par détournement des moyens de production lors de grève ou d'occupations d'usine ". C'est ainsi que paraît dans la presse, en 1998, une annonce destinée à collecter ces objets dans le cadre de la préparation de l'exposition de Jean-Luc Moulène à La Galerie, centre d'art situé à Noisy-Le-Sec ${ }^{6}$. Les 
circonstances et les acteurs de leur production sont donc particuliers. L'histoire des conflits sociaux et, plus précisément des grèves, est émaillée d'épisodes iconoclastes où l'usine devient un théâtre d'activités qui, d'ordinaire, relèvent généralement du domaine du " hors travail ". Comme l'écrit Stéphane Sirot ${ }^{7}$, " puisqu'elle rompt avec le commun, la grève s'accompagne d'un cortège de pratiques, de faits et gestes qui sortent de l'ordinaire, au sens où ils s'inscrivent dans des modalités de transgression de la norme sociale. Surtout quand elles durent, les grèves ouvrent la voie à des modes de protestation, de communication, d'extériorisation qui contribuent à construire la solidarité et l'identité d'un groupe ".

\section{Occupations}

\section{Figure 5}

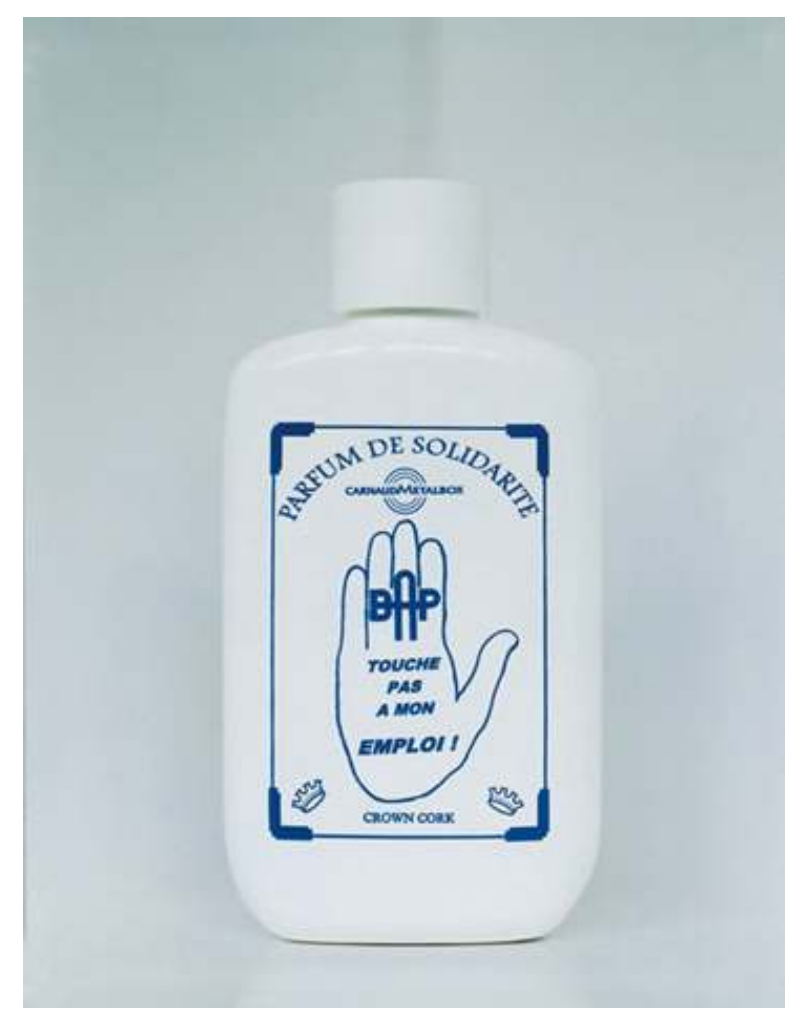

Parfum de solidarité. Flacon en plastique blanc moulé. Bourgogne Applications Plastiques, France à Chevigny-Saint-Sauveur (Côte-d'Or) (filliale de Carnaud Metalbox, maison-mère : Crown Cork). Courtesy galerie Chantal Crousel, Paris

(c) Jean-Luc Moulène - ADAGP, 1999.

Parmi ces modes d'intervention, l'occupation des locaux et les rituels qui l'accompagnent créent les conditions d'une remise en question des fonctionnements traditionnels de l'entreprise. La fonction productive est remplacée, pour un temps, par des événements festifs pendant lesquels s'expriment, dans un temps nouveau, la prise de possession de l'usine par le personnel d'exécution. On assiste notamment à un renversement de sa hiérarchie traditionnelle: la direction étant placée en suspens, dans un hors-jeu temporaire, l'ouvrier devient concepteur de l'objet, maitre d'œuvre unique de sa fabrication. En résumé, il s'agit à la fois de faire entrer dans l'usine des éléments de convivialité et de solidarité qui en sont habituellement exclus ou atténués, et d'autre part 
d'utiliser de façon détournée le dispositif de production: la machine va être réglée de façon particulière (pour fabriquer de petites quantités), l'outil utilisé avec un surplus d'inventivité, afin de donner à l'objet voulu une forme inhabituelle ${ }^{8}$ (fig. $\mathbf{n}^{\circ} 5$ ). Les techniques de fabrication sont utilisées pour un autre but que celui visé d'ordinaire (ce qui peut apparenter ce phénomène d'objet de grève à celui de la perruque) ${ }^{9}$.

Figure 6

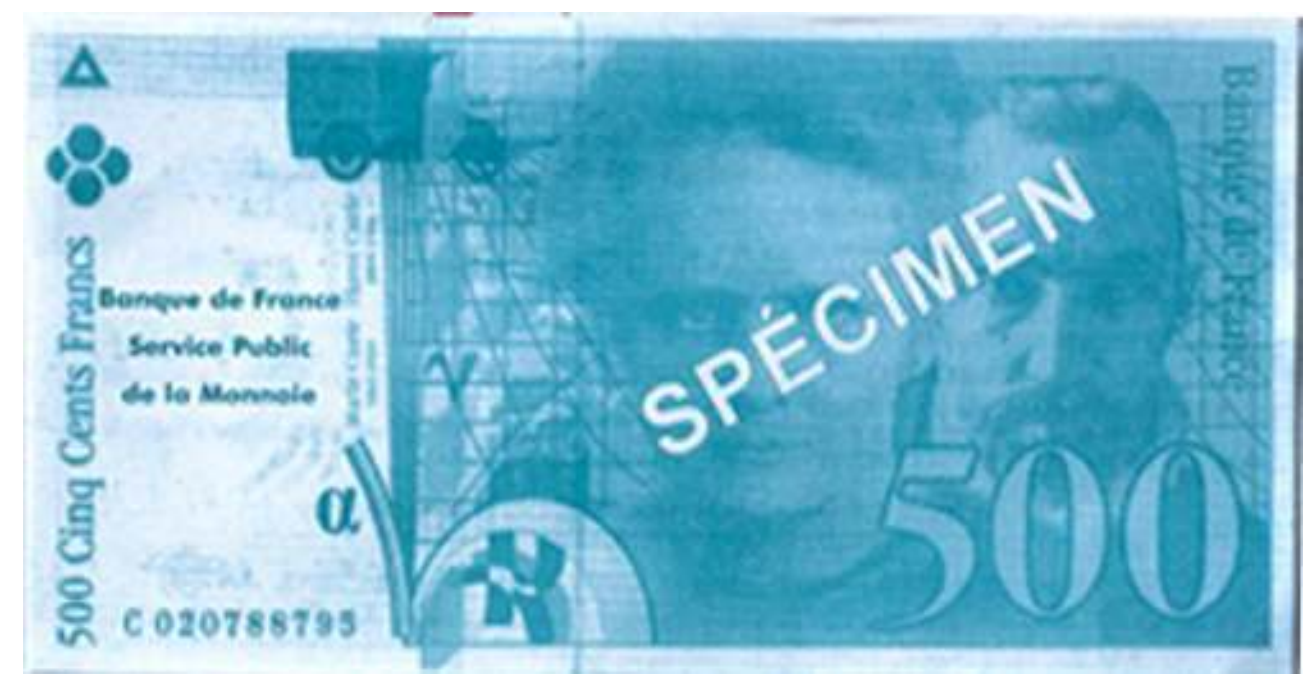

Marie et Pierre Curie, le billet. Imprimerie de la Banque de France sur le site de Chamalières (Puy-deDôme), 1998. Fac-similé offset vert du billet de 500 FF, n C 020788795. $17 \mathrm{~cm}$ x 8,8 cm. Barrant les visages de Marie et Pierre Curie le mot SPECIMEN. Courtesy galerie Chantal Crousel, Paris

(c) Jean-Luc Moulène - ADAGP, 1999.

11 La solidarité peut se révéler ou émerger à nouveau dans ces moments-là, avec plus de force et de constance que d'ordinaire. Le rapport individuel quotidien tend à laisser place à une complicité toute collective. Il faut dire que " la fête n'est pas que joie, elle est aussi un exutoire, celui de tensions accumulées dans le quotidien de la vie productive "10. Dans ces moments-là, la production d'objets est un des signes d'appropriation de l'espace de production. On fait montre d'un savoir-faire technique, mais pas seulement : il y a acte de création car l'objet fabriqué n'est pas un objet standard, mais une réinterprétation, une modification, voire une véritable nouveauté1 ${ }^{11}$ (fig. $\mathbf{n}^{\circ}$ 6). 


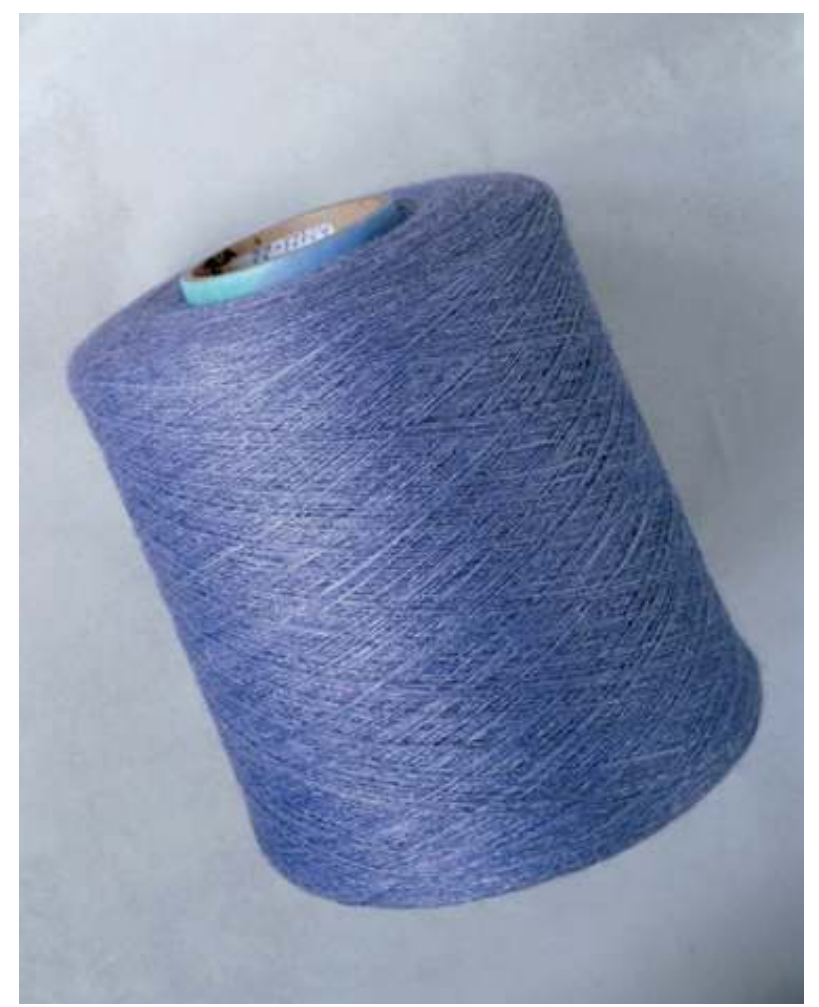

La Bobine Novacore. France, Ateliers Roannais de Construction Technique (ARCT), Roanne (Loire). Création 1980. Diamètre 4,5 cm, hauteur 5,5 cm. Bleu. Références : NM 24/2 - AMC PA 78/20 FT $19 \%$ Laine 81 \% - A 2123-22. (Collection " La Vie en Pull ", Roanne). Courtesy galerie Chantal Crousel, Paris (c) Jean-Luc Moulène - ADAGP, 1999.

Les objets permettent de valoriser le travail et le savoir-faire de métier, de démontrer aussi que l'usine est viable et que l'innovation ou le travail de qualité sont possibles. Comme l'écrit encore Stéphane Sirot ${ }^{12}$, “l'occupation [...] prend aussi l'allure, au moins symboliquement, d'une manière d'appropriation du lieu de production qui marque le refus de le voir mourir. Parfois, les grévistes cessent brièvement le travail avant de remettre les machines en route. Ainsi les ouvriers [...] qui, après le dépôt de bilan de leur usine, l'occupent et reprennent la fabrication [...] qu'ils cèdent en échange d'une solidarité financière ${ }^{113}$ (fig. $\mathbf{n}^{\circ}$ 7).

\section{Diffusion}

13 S'il y a valorisation du savoir-faire ouvrier au-delà des frontières traditionnelles de son emploi, l'objet de grève permet aussi de communiquer autour du conflit. Car, destiné à être diffusé à plus ou moins grande échelle (souvent une diffusion de proximité, attachée à un territoire donné), l'objet est un vecteur concret pour faire connaître la lutte et pour la financer. Cette action constitue souvent une des facettes d'initiatives plus larges: manifestations de rue, opérations portes ouvertes dans l'usine, etc. Par la vente, on rallie des solidarités externes à l'entreprise: habitants, centrales syndicales, organisations politiques, médias, etc. Loin de l'objet industrialisé dont il détourne le processus normal de fabrication, l'objet de grève est un objet militant qu'on fabrique et achète pour 
soutenir la lutte. Les objets de grève apparaissent aujourd'hui comme les témoins d'une forme de manifestation sociale qui a quasiment disparu.

Figure 8

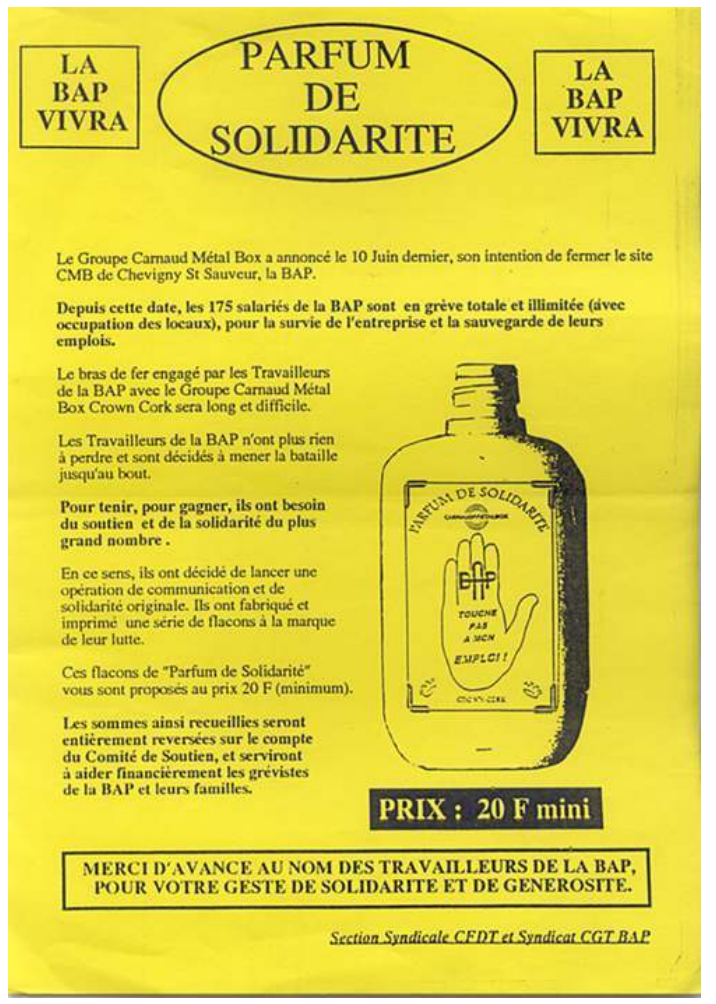

Parfum de solidarité. La BAP vivra (tract section syndicale CFDT et Syndicat CGT BAP). Provenance : Archives Nationales du Monde du Travail, fonds Jean-Luc Moulène (cote 2003 025)

(c) Jean-Luc Moulène - ADAGP, 1999.

Même s'il travaille sur de nouveaux sujets ${ }^{14}$, Jean-Luc Moulène poursuit sa collecte, mais avec difficulté : “je reçois encore des informations, mais on produit moins d'objets de grève. Dans les années 1980, le produit est le mode de communication dominant : comme une grève a besoin de se faire connaître, on fabrique des objets. Aujourd'hui, le spectacle est devenu le mode de communication principal, ce qui explique que les conflits du travail se concluent parfois par des prises d'otage ou des menaces de pollution de site" (fig. $\mathbf{n}$ - 8). On se souvient par exemple des luttes dans les entreprises Metaleurop ${ }^{15}$ ou Cellatex. Les modes de militance syndicales aussi ont évolué : à l'ère du virtuel, le tract est moins présent, la transmission des messages passe de plus en plus par le Web ou la messagerie électronique. On assiste à une certaine dématérialisation de la lutte, ce qui explique pour partie que l'on fabrique moins d'objets de grève.

\section{Objets d'art}

15 Jean-Luc Moulène travaillait depuis plusieurs années sur les questions touchant à la façon dont les images apparaissent et sont construites dans nos sociétés contemporaines quand il a eu connaissance de l'existence de ces objets singuliers. Sa réflexion s'articulait alors autour de trois modalités qu'il énonce ainsi: "conditions de production, mode d'apparition et diffusion des images ${ }^{{ }^{16}}$ (fig. $\mathbf{n}^{\circ} \mathbf{9}$ ). 
Figure 9

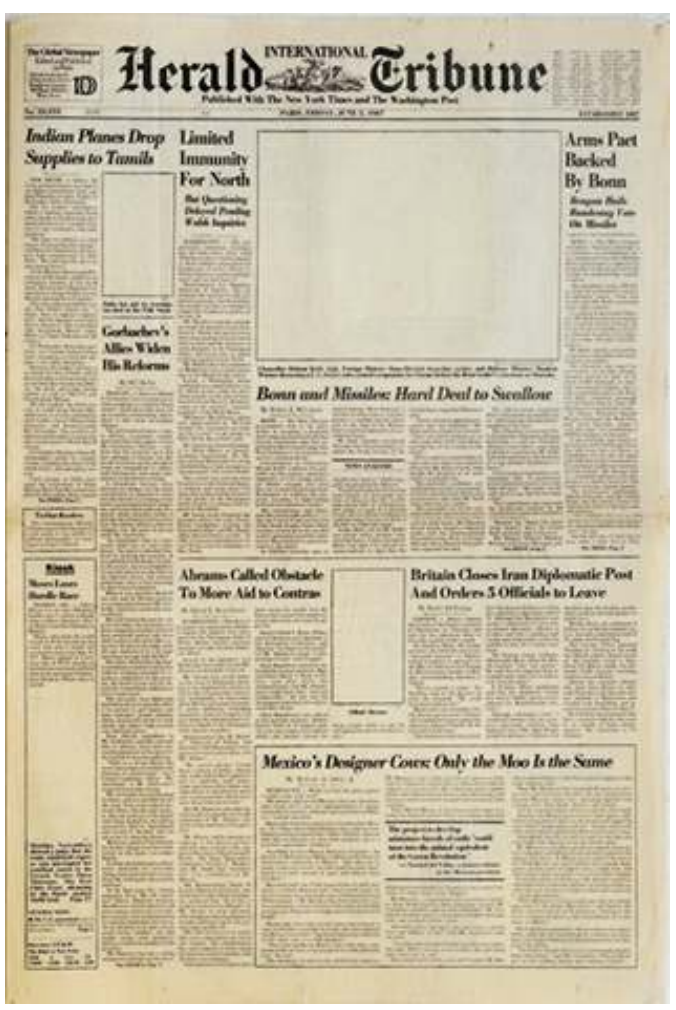

International Herald Tribune. $n^{\circ} 32434$ du vendredi 5 juin 1987, Paris. Un retoucheur ayant subi une compression d'horaires se mit en grève de retouche et, par solidarité, les photograveurs ne gravèrent point. Courtesy galerie Chantal Crousel, Paris

(c) Jean-Luc Moulène - ADAGP, 1999.

Etudiant ces trois moments et leur corrélation, il isole les objets de leur environnement habituel pour mieux interroger leur nature et leur signification. Il commence par collecter un numéro du journal américain The Herald Tribune paru le vendredi 5 juin 1987 : " dès 1983, je photographiais des panneaux publicitaires vides. Pour Le Herald, la grève d'un gars avait produit ce manque visuel fort ". Puis lui revient en mémoire un paquet de Gauloises rouges dont il avait eu connaissance quelques années auparavant ${ }^{17}$ (fig. $\mathbf{n}^{\circ} \mathbf{1 0}$ ). 


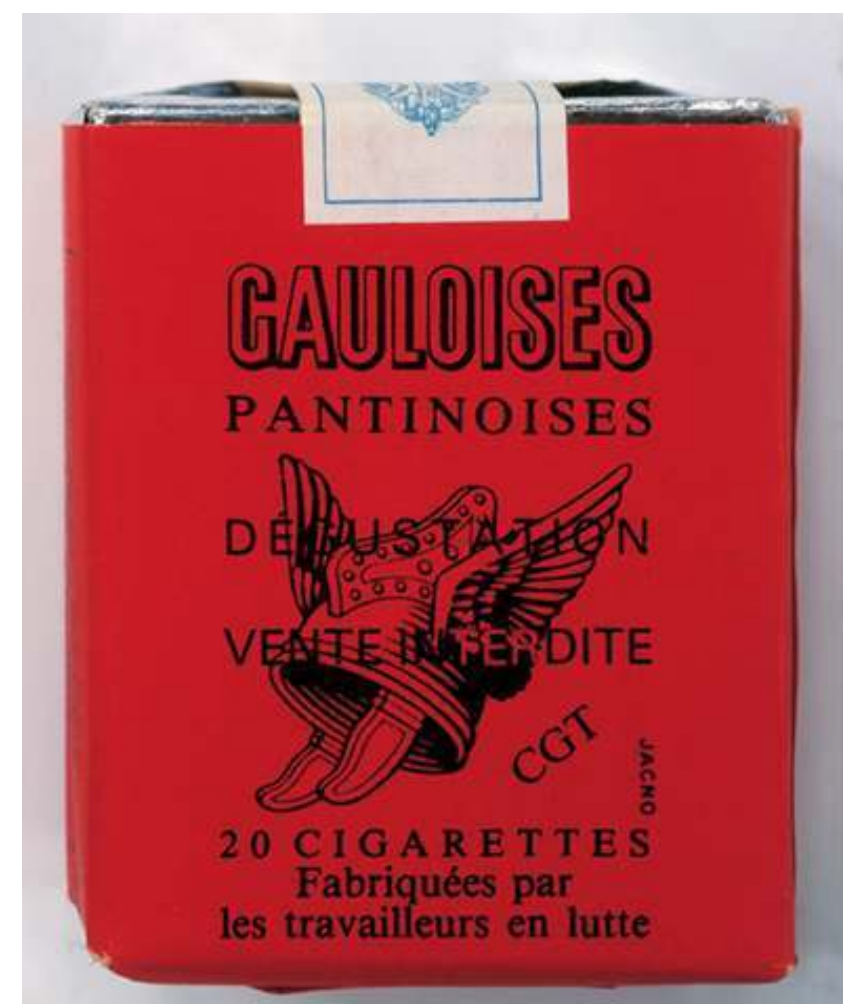

La Pantinoise. Paquet de cigarettes rouge. France, usine des tabacs de Pantin (Seine-Saint-Denis), Seita, 1982-1983. Courtesy galerie Chantal Crousel, Paris

(c) Jean-Luc Moulène - ADAGP, 1999.

Ces deux objets mis côte à côte constituent un début de collection qu'il devait poursuivre à l'occasion d'un projet d'exposition au Centre d'art de Noisy-le-Sec. Il débute alors la recherche d'autres objets. L'actualité lui offre des opportunités à plusieurs reprises car les années 1980 sont émaillées de fermetures de grandes entreprises et de conflits sociaux. Il prend contact avec des grévistes, des fédérations syndicales, rencontre divers acteurs du monde du travail. Une fois collecté, l'objet est photographié en atelier, à la lumière du jour, sur fond neutre. Jean-Luc Moulène raconte: "il s'agit de rendre le poids et la matière de l'objet car c'est sur cette matière que les ouvriers ont travaillé. Je fabrique un objet photographique, pas une photographie, qui a tous les caractères d'un produit industriel ". Ce processus emporte l'adhésion des ouvriers pour lesquels le travail de l'artiste valorise leur savoir-faire professionnel et embellit, tout autant qu'il rappelle ou commémore, leur vie militante. Simultanément, les objets photographiques de Jean-Luc Moulène séduisent le monde de l'art. Le Centre Georges Pompidou les achète et les fait entrer dans ses collections permanentes. "Il y a là une ambiguïté. Dans mon esprit, cette œuvre est un lieu de conflit, pas un lieu pacifié. Beaubourg achète les photographies, ce qui représente l'assomption en art d'un objet manifeste. D'une certaine manière, le contenu subversif de l'objet de grève est ainsi apaisé".

Nous sommes donc en présence d'un dispositif complexe : les objets de grève sont des altérations d'objets industriels transformés ensuite en œuvres plastiques. Cette mutation en chaîne permet de faire connaître les luttes sociales qui ont donné naissance aux objets et de faire le lien entre travail et art, industrie et musée. Dans un entretien donné à la 
revue électronique Inventaire/Invention, Jean-Luc Moulène énonce l'impact social et symbolique que l'exposition des objets a produit sur ses visiteurs: "il semblerait que cette exposition ait assumé un désir de grève qui existe dans pratiquement toutes les couches de la population mais qu'on n'ose pas mettre en jeu au sein de sa propre entreprise. Que la fonction de grève soit assumée de façon cathartique via l'art est un fait que je n'avais pas prévu. J'aurais préféré que l'exposition mette les gens en grève plutôt que de répondre à leur désir passif de grève. Il est vrai que depuis quelques années, ce phénomène se reproduit régulièrement où l'on voit la population se réjouir lorsqu'un mouvement social éclate, lorsque la grève est faite. Un plaisir naît ainsi de ces grèves par délégation et l'exposition a fait naître un plaisir de cette sorte".

\section{Objets d'archive}

19 En 2000 puis 2003, Jean-Luc Moulène confie aux Archives nationales des objets de grève ${ }^{18}$ collectés auprès d'ouvriers, de militants syndicaux ou associatifs " afin de séparer ce qui fait l'ambiguïté du monde de l'art et de sa circulation, du travail d'archiviste". Les œuvres photographiques, quant à elles, sont acquises par le Musée national d'art moderne (Centre Georges Pompidou) et entrent dans les collections nationales. Aux Archives Nationales du Monde du Travail, les objets de grève sont re-conditionnés, décrits, puis prennent place dans les magasins d'archives. Même si le Centre collecte essentiellement des archives papier (correspondance, pièces administratives, registres comptables et du personnel, documentation technique...), il n'est pas rare que les fonds se composent de supports composites: plans ou cartes de grand format, supports photographiques, audiovisuels ou sonores, etc. Les objets en trois dimensions représentent une faible masse parmi les 35 à 40 kilomètres d'archives conservés actuellement à Roubaix. On en retrouve notamment dans les fonds d'architectes (Couëlle, Simounet, Sarfati, etc.) qui, outre les dossiers administratifs et les plans en rouleaux, comprennent aussi des maquettes de projets ou pré-projets. Mais il arrive aussi qu'au hasard d'une collecte les archivistes soient amenés à sauvegarder des objets emblématiques de l'activité d'une entreprise dont ils accueillent les archives classiques. Ainsi, en 2005, une compagnie d'assurances, en même temps qu'elle faisait don à l'État de ses archives administratives, cédait des objets publicitaires ou de bureau en rapport direct avec son activité : badges, pins, coupes et médailles récompensant les meilleurs équipiers, cadeaux publicitaires destinés aux clients, mais aussi anciennes machines à écrire ou à calculer, petit meuble métallique de classement, etc. Ces objets devraient former à terme, avec ceux issus d'autres fonds, un ensemble cohérent qui sera susceptible d'être exposé dans les espaces culturels du Centre.

20 Ce traitement des objets, outre qu'il s'applique à une typologie de documents nous renseignant utilement sur leur producteur, est en adéquation avec la loi du 3 janvier 1979 sur les archives, reprise dans le Code du patrimoine en 2004, qui ne réduit pas la notion d'archives à un type particulier de support. D'ailleurs, en terme de traitement de fonds aux formes hétérogènes, il est recommandé19 " " dans le cas où un document se décompose en plusieurs éléments sur des supports physiques différents, de ne pas se laisser influencer et d'archiver le document comme un tout dont les parties auront des règles de conservation physique un peu différentes". Pour autant, tout document n'est pas une archive. Ce qui constitue le document d'archives, c'est tout document à partir du moment 
où “il est appréhendé dans sa dimension de trace d'une activité, de preuve et de témoignage d'un acte ou d'un événement que l'on peut localiser et dater "20.

21 Le cas des objets de grève ne diffère guère de cette règle. Cependant, même si les objets de grève ont été, au final, collectés et rassemblés par la même personne (Jean-Luc Moulène), ils ne proviennent pas d'un producteur unique (plusieurs conflits dans plusieurs entreprises). Ce sont plutôt les conditions dans lesquelles ils ont été produits qui créent leur unité. Ils permettent ainsi d'enrichir les fonds syndicaux ou militants qui, pour certains, comportent des pièces de même nature : panneaux d'exposition présentant des luttes sociales, drapeaux utilisés pendant des manifestations, etc. Le don de Jean-Luc Moulène s'enrichira peut-être, dans les prochains mois, des dossiers de recherche que ce dernier avait constitués lorsqu'il réalisait son travail de contact et de repérage des objets : des dossiers par entreprise comprenant notes de travail, coordonnées des contacts, tracts syndicaux, à l'exclusion de ses carnets d'artiste qui resteront sans doute en sa possession. En effet, on peut considérer que ceux-ci témoignent plus du processus intime de production des objets photographiques et de la réflexion qui l'entoure, que des objets de grève eux-mêmes.

\section{Objets sensibles}

Pour les sciences sociales, les objets constituent une source progressivement prise en compte par les chercheurs. Noëlle Gérôme les désigne comme des "archives sensibles "21 nommées ainsi " puisque, hors de la médiation de l'écriture, elles apportent directement pour les objets, indirectement pour les images, le témoignage de l'appréhension sensorielle des choses, des individus ou de la situation, par la vision, le toucher, la sensibilité kinesthésique". Les approches artistique et archivistique de l'objet s'enrichissent d'une approche anthropologique par laquelle, au-delà de ses caractéristiques matérielles, l'objet est considéré comme porteur de la culture d'un groupe particulier, de ses rites, manières de faire, visions du monde. Comme l'ajoute Noëlle Gérôme ${ }^{22}$, “il semble bien qu'une activité symbolique à l'œuvre dans les sites industriels trouve dans la production d'objets ou dans l'adoption d'objets symboliques, son expression la plus forte et la plus constante. Il s'agit [...] de l'adoption et de l'appropriation signifiante d'objets dans lesquels se reconnaissent et s'affirment des groupes qui cherchent à faire reconnaître leur légitimité et celle de leur discours dans l'enceinte primitivement interdite des usines [...]. Des objets vestiges aux objets symboles, il y a le sens d'une demande constitutive d'histoire, les difficultés à ordonner une mémoire afin de la constituer et surtout de la restituer ".

Les objets de grève constituent des traces exprimant la capacité d'un groupe à organiser sa résistance dans la grève (en s'autogérant et en finançant sa subsistance) et à dépasser le conflit en demeurant producteur. Ils sont révélateurs d'une culture à la fois ouvrière et militante, comme le montrent la couleur ou les slogans des objets : cris de colère, appels à l'aide, mais aussi continuité dans la production des objets ${ }^{23}$ (fig. $\left.\mathbf{n}^{\circ} \mathbf{1 1}\right)$. 
Figure 11

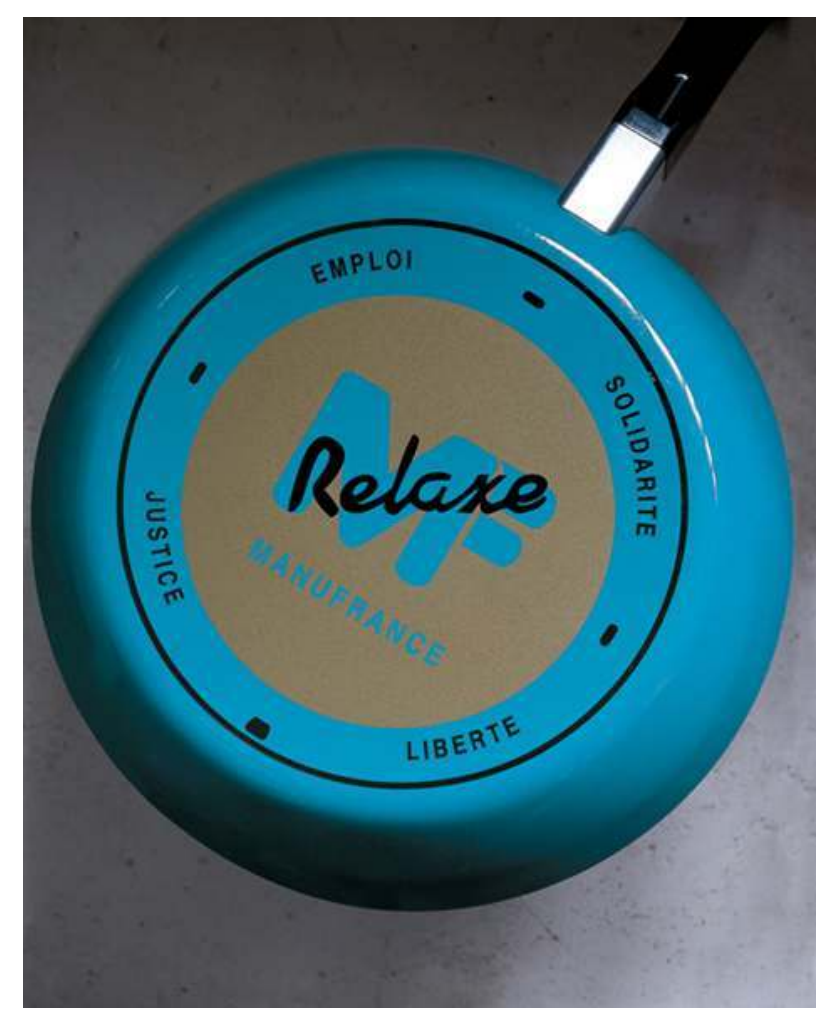

La poêle des 17 de Manufrance. Manufrance, Saint-Etienne (Loire), 1993. Poêle d'un diamètre de 20 $\mathrm{cm}$, fond de chauffage vert avec en son centre un cercle doré sur lequel s'inscrivent MF Manufrance et Relaxe en noir. (Collection Comité d'entreprise de la société Téfal). Courtesy galerie Chantal Crousel, Paris

(C) Jean-Luc Moulène - ADAGP, 1999. chose est bousculé dans l'ordre des choses. On peut remarquer aussi que le type d'objets
ainsi produits n'est pas anodin. Ils forment un patchwork, mais ont tous, de près ou de loin, rapport avec nos besoins vitaux : se vêtir, se déplacer, se nourrir, etc. De la cigarette, Jean-Pierre Burdin ${ }^{24}$ écrit justement: “Qu'est-ce qui propage le mieux les idées et les affects que la cigarette [...] proposée au compagnon de travail ? Le tabac tue, oui, mais alors il faudra bien inventer autre chose pour passer le feu, partager une colère rentrée, libérer un sanglot". Il y a, dans l'assemblage de ces objets, une sorte "d'ethnologie française ${ }^{25}$, une manière de percevoir et de comprendre notre société dans sa globalité et dans son mode de fonctionnement.

Se dessine ainsi un visage inattendu du monde industriel et de son patrimoine : le passé de l'industrie se compose aussi de ces objets marginaux, cachés derrière les murs parfois monumentaux des usines, invisibles dans le flot des produits vantés par la publicité et consommés en masse. Ils apparaissent dans une sorte de désorganisation du travail standardisé, dans les interstices d'un rythme de production soudain stoppé, laissant place à l'utopie d'une gestion d'entreprise et d'une vie sociale différentes. Car ces objets de grève sont avant tout des objets politiques qui racontent une résistance et l'assomption d'une forme de culture d'ordinaire dominée et bâtie autour des savoir-faire de métier, de la solidarité propre à une même condition sociale et à des intérêts convergents, de l'inventivité aussi que le taylorisme et ses prolongements contraignent si souvent. A côté 
des bâtiments et machines de l'industrie, ces objets témoignent bien de la réalité d'un "patrimoine industriel social" qui peine parfois à émerger malgré les nombreuses initiatives visant à construire ça et là des récits mémoriels de vies au travail. En l'occurrence, c'est une intervention artistique et militante à la fois, liant le respect pour les hommes en lutte et la volonté de faire connaître leur qualité par la médiation de l'art, qui aura permis que ces traces de l'histoire sociale puissent parvenir jusqu'aux centres d'art ou d'archives et au grand public.

\section{NOTES}

1. Les Archives Nationales du Monde du Travail (anciennement Centre des Archives du Monde du Travail, CAMT), service à compétence nationale, est l'un des cinq centres d'Archives nationales (ministère de la Culture et de la Communication, Direction des archives de France). Il est spécialisé dans le traitement d'archives d'entreprises, d'associations et de syndicats. Inauguré en 1993, il est implanté à Roubaix (Nord) dans l'ancienne filature de coton Motte-Bossut.

2. T-shirt en coton, sérigraphie bleu blanc rouge noir représentant Marianne sur fond de BastilleUsine "même combat" avec le slogan "vivre et travailler autrement"; Colombe et "La Vie en Pull " au-dessus des deux forteresses assiégées. Prix unitaire : adulte 40 frs, enfant 35 frs. Étaient également vendus le Saint-Just (Le bonheur est toujours une idée neuve), les 3 colombes et bien d'autres. Aujourd'hui, les ouvrières de l'Association vendent leur stock pour régler leur passif, le loyer, les frais de justice. En effet, après la cour de cassation, c'est la cour européenne de justice qui instruit "l'Affaire". Les produits sont vendus avec l'aide syndicale et associative à des prix minorés, dans le refus du chômage, de la précarité et des délocalisations (@Viviane de Tapia, 1999). Provenance de l'objet photographié : Archives Nationales du Monde du Travail, fonds JeanLuc Moulène (cote 2003025 004).

3. ASSOCIATION QUÉBÉCOISE POUR LE PATRIMOINE INDUSTRIEL. L'objet industriel. Actes du congrès, Hull, 3 et 4 mai 1991, janvier 1992.

4. Souliers couleurs "foot" rouge et noir, longueur $8,5 \mathrm{~cm}$, largeur voûte plantaire $3,5 \mathrm{~cm}$, cuir pleine peau. Les souliers ont été réalisés pendant l'occupation des usines, à partir de novembre et décembre 1995 ainsi que dans les moments forts, afin de les vendre les 1er mai 1996 et 1997, lors de rencontres sportives, de manifestations et d'actions. La Société Myrys a été reprise par un groupe anglais en février 1998. Les revendications des travailleurs telles que la production de 10000 paires de souliers par semaine, et la rénovation de 40 magasins Myrys n'ont, à ce jour, pas été satisfaites (OViviane de Tapia, 1999). Provenance de l'objet photographié: Archives Nationales du Monde du Travail, fonds Jean-Luc Moulène (cote 2003025 009).

5. Sauf indication contraire, les citations de cet article sont extraites d'un entretien avec Jean-Luc Moulène qui a eu lieu à son domicile parisien le 28 juin 2006. Cet enregistrement s'insère dans un programme de constitution d'archives orales et audiovisuelles engagé depuis peu par les Archives Nationales du Monde du Travail et dont le but consiste à recueillir les témoignages de personnes ayant donné ou déposé des archives au Centre, à titre privé ou comme membre d'une entreprise, d'une association ou d'un syndicat.

6. L'exposition Vingt-quatre objets de grève présentés par Jean-Luc Moulène a eu lieu à La Galerie, Noisy-le-Sec (Seine-Saint-Denis), du 20 mai au 13 juillet 1999. Elle était accompagnée d'un catalogue du même nom. Elle a été suivie d'une autre exposition organisée en 2000 à 
Forbach (Moselle) et intitulée Trente-neuf objets de grève présentés par Jean-Luc Moulène. En mars 2003, les objets de grève ont été exposés au congrès de la CGT : à cette occasion, un encart a été publié dans le périodique La Nouvelle Vie Ouvrière : MOULÈNE, Jean-Luc, DE TAPIA, Viviane, GÉRÔME, Noëlle. Quarante objets de grève présentés par Jean-Luc Moulène. La Nouvelle Vie Ouvrière, 2003, supplément au numéro 3057 du 24 mars 2003 publié à l'occasion du 47ème congrès de la CGT.

7. SIROT, Stéphane. La grève en France, une histoire sociale (XIXe-XXe siècle). Paris : Editions Odile Jacob, 2002, p. 143.

8. Flacon d'une hauteur de $7,5 \mathrm{~cm}$, plastique blanc moulé, étiquette " sérigraphiée " (gamme de 6 couleurs) comportant sur une face : "BAP vivra S.0.S. 175 emplois", et sur l'autre "Parfum de solidarité ", et sur le dessin d'une main ouverte : "BAP Touche pas à mon emploi! ” Le conflit a commencé le 10 juin 1996 et s'est terminé le 29 septembre 1996. Le prix de vente du flacon était de $20 \mathrm{~F}$ minimum. 10000 flacons ont été vendus en 8 semaines. Les 175 salariés de la BAP ont été en grève illimitée avec occupation des locaux ; ils fabriquèrent " la plus grosse bouteille plastique en PVC du monde" (hauteur 6,82 $\mathrm{m}$, diamètre 1,79 $\mathrm{m}$, nombre de flacons pour la composer: 6865 , poids de $307 \mathrm{~kg}$ ) ; elle n'intégrera pas le livre des Records Guinness (bouteille composite). Le groupe allemand Alpla reprend l'entreprise et 55 emplois sous le nom de Euroflaco (@Viviane de Tapia, 1999). Provenance de l'objet photographié : Archives Nationales du Monde du Travail, fonds Jean-Luc Moulène (cote 2003025 007).

9. Voir le site : http://fr.wikipedia.org/wiki/Travail_en_perruque.

10. SIROT, Stéphane. La grève en France, une histoire sociale (XIXe-XXe siècle). Paris : Editions Odile Jacob, 2002, p. 150.

11. Fac-similé monochrome vert. Au dos du billet : revendications du personnel et des syndicats CFDT, CGT, FO, SI\&C, SNA - agissant auprès des élus et des usagers pour défendre la qualité du Service Public: "Contre l'abandon du Service Public de la Monnaie Contre les risques de désertification locale. Le Gouverneur TRICHET a déjà supprimé 800 emplois à la Fabrication des billets, au prétexte d'alignement sur les coûts de production européens. Sa stratégie implique, d'ores et déjà, la suppression d'une centaine de caisses, d'un millier d'emplois et à moyen terme la fermeture des succursales correspondantes. " Ce billet vert, largement distribué pendant les grèves, revendique la conservation des Caisses et le maintien du réseau de la Banque de France (@Viviane de Tapia, 1999). Provenance de l'objet photographié : Archives Nationales du Monde du Travail, fonds Jean-Luc Moulène (cote 2003025 001).

12. SIROT, Stéphane. La grève en France, une histoire sociale (XIXe-XXe siècle). Paris : Editions Odile Jacob, 2002, p. 123.

13. ARCT, Ateliers Roannais de Construction Technique, le conflit : création des ARCT en 1922 par Elysée Crouset, 1956 création de la machine FT1 (Fausse Torsion), 1974 les ARCT emploient 1850 travailleurs et 800 personnes en sous-traitance. En 1975, la crise éclate (implantation des industries dans les pays à main-d'œuvre à moindre coût); dix années de luttes s'ensuivent. La filière du textile artificiel devient un enjeu national : mobilisations syndicales, manifestations, grèves. En 1980, les travailleurs de l'ARCT inventent le Novacore (fil à âme nylon gainé alliant qualités de la fibre - laine, lin, etc. - à la robustesse du synthétique) ainsi que la machine RS 200 pour sa production $(210 \mathrm{~m} / \mathrm{mn})$. C'est une avancée technique considérable. 1981 : menaces de vente du procédé Novacore au Japon et colère des syndicats. 1985 : plan de reprise proposé par B. Terrat (sans plan social) refusé en bloc. Le brevet Novacore est vendu à la Suisse. Juin 1985 : liquidation des biens des ARCT sans continuation de l'activité (@Viviane de Tapia, 1999). Provenance de l'objet photographié : Archives Nationales du Monde du Travail, fonds JeanLuc Moulène (cote 2003025 006).

14. Jean-Luc Moulène a présenté récemment plusieurs expositions dont une consacrée à la série intitulée Les filles d'Amsterdam (Jeu de Paume, Paris, 2005) et une autre aux statuettes conservées au Musée du Louvre et intitulée Le Louvre (Paris, 2006). 
15. Le fonds d'archives de Metaleurop Nord est entré aux Archives Nationales du Monde du Travail en 2006 et sera prochainement accessible aux chercheurs.

16. Le Herald Tribune publiait en une cet encadré : “À nos lecteurs : suite à une grève d'une partie du personnel, le Herald Tribune regrette d'être dans l'impossibilité de publier les photographies illustrant la plupart des articles de l'édition d'aujourd'hui "; par exemple: "Saul Bellow: les gens ordinaires peuvent encore assister à une représentation du "Roi Lear " et pleurer " s'inscrit sous un carré blanc avec le nom du photographe (Thomas Victor) en page $18 \mathrm{du}$ journal (CViviane de Tapia, 1999). Provenance de l'objet photographié : Archives Nationales du Monde du Travail, fonds Jean-Luc Moulène (cote 2003025 001).

17. Paquet rouge $5,8 \mathrm{~cm}$ de large, $7 \mathrm{~cm}$ de long, profondeur $2,3 \mathrm{~cm}$. Inscription sur face reproduite: “ "Gauloises pantinoises. Dégustation. Vente interdite. CGT. 20 cigarettes fabriquées par les travailleurs en lutte. Jacno". Sur l'autre face: “Gauloises, usine des tabacs de Pantin, Seita, Jacno, occupée depuis le 23.2.82 pour le redémarrage, contre la fermeture de l'établissement". Sur l'une des tranches : “Taxes prélevées $74 \%: 2,88$ frs. Prix chez le buraliste 3,90 frs". Les travailleurs de la manufacture des tabacs de Pantin, occupant les locaux pour le redémarrage de l'usine depuis le 23 février 1982, décident de la remise en route de l'entreprise en créant le 11 juin 1982 “La Pantinoise”. Le paquet de Gauloises rouge était offert avec le bon de soutien à $5 \mathrm{~F}$. Au moment où le conflit s'est calmé, la majorité des paquets restants a été détruite (Collection Paul Girard, CGT Grenoble) (@Viviane de Tapia, 1999). Provenance de l'objet photographié : Archives Nationales du Monde du Travail, fonds Jean-Luc Moulène (cote 2003025 008).

18. Quarante objets de grève sont conservés aux Archives Nationales du Monde du Travail sous le numéro d'entrée 2003 025. Ils ont d'abord été déposés par Jean-Luc Moulène avant que ce dépôt ne soit transformé en don en 2006. D'autres objets de grève provenant de la Société coopérative anonyme La Vie en Pull sont référencés au Archives Nationales du Monde du Travail sous le numéro d'entrée 2002026014.

19. ASSOCIATION DES ARCHIVISTES FRANÇAIS. Abrégé d'archivistique, principes et pratiques du métier d'archiviste. Paris : Association des archivistes français, 2004, p. 53.

20. ASSOCIATION DES ARCHIVISTES FRANÇAIS. Abrégé d'archivistique, principes et pratiques du métier d'archiviste. Paris : Association des archivistes français, 2004, p. 51.

21. GÉRÔME, Noëlle (sous la direction de). Archives sensibles, images et objets du monde industriel et ouvrier. Cachan : Editions de l'ENS-Cachan, 1995, p. 11.

22. GÉRÔME, Noëlle (sous la direction de). Archives sensibles, images et objets du monde industriel et ouvrier. Cachan : Editions de l'ENS-Cachan, 1995, p. 157.

23. Sur le pourtour de la poêle, en lettres noires on peut lire: “Emploi, Solidarité, Liberté et Justice ". En 1984, 17 syndicalistes et gestionnaires tentent de sauver Manufrance et de préserver l'emploi en créant une coopérative ouvrière. Ils sont dans l'obligation de déposer le bilan le 4 avril 1985. Le 9 juillet 1992, les 17 “Manu” sont condamnés par le Tribunal Correctionnel de Saint-Etienne ; 36 ans d'emprisonnement - dont 22 ans avec sursis - 36400000 francs d'amendes sont distribués aux 10 syndicalistes, 5 gestionnaires et 2 experts comptables. En mars 1993, la poêle Manufrance est créée par la CGT en soutien financier aux condamnés, c'est un appel à la relaxe des 17 en vue du procès qui aura lieu le 31 mars 1993 et qui se conclut par la relaxe de 14 personnes et l'amnistie des 3 autres. (Collection comité d'entreprise de la Société TEFAL) (CViviane de Tapia, 1999).

24. MOULÈNE, Jean-Luc, DE TAPIA, Viviane, GÉRÔME, Noëlle. Quarante objets de grève présentés par Jean-Luc Moulène. La Nouvelle Vie Ouvrière, 2003, p. 9, supplément au numéro 3057 du 24 mars 2003 publié à l'occasion du 47ème congrès de la CGT.

25. Entretien avec Jean-Luc Moulène du 28 juin 2006. 


\section{RÉSUMÉS}

Les objets de grève sont des produits fabriqués en petite série par des ouvriers à l'occasion de conflits $\mathrm{du}$ travail. Ils revêtent des formes et fonctions multiples: ne répondant pas aux standards de fabrication, ils servent notamment à populariser et financer la grève. A partir des années 1980, ces objets ont été collectés et photographiés par Jean-Luc Moulène, artiste contemporain. Les œuvres qui résultent de ce processus ont été plusieurs fois exposées. Les objets eux-mêmes ont été donnés à l'État et sont conservés aux Archives Nationales du Monde du Travail (Roubaix).

Strike objects are objects made in small series by workers during industrial conflicts. They have various functions and forms. They do not fit into ordinary industrial production, but are often particularly useful for popularising or financing a strike. From the 1980s, many of these objects have been collected and photographed by Jean-Luc Moulène, a contemporary artist. The results of his work have been exhibited on several occasions and the objects themselves have been given to the State. They are held today at Roubaix, at the national archive depot for the archives of the world of work (Archives Nationales du Monde du Travail).

\section{INDEX}

Keywords : strike, strike objects, Jean-Luc Moulène, archives of the world of work, Roubaix, worker, militancy, industry, art Striking objects, heritage of militancy

\section{AUTEUR}

\section{JEAN-CHARLES LEYRIS}

Ethnologue. Responsable du développement culturel, de l'iconographie et de l'audiovisuel aux Archives Nationales du Monde du Travail (Roubaix). jean-charles.leyris@culture.gouv.fr 